This is the post-print author manuscript of

\title{
Performance of GaAs Nanowire Array Solar Cells for Varying Incidence Angles
}

\author{
Omid Madani Ghahfarokhi, Nicklas Anttu, Lars \\ Samuelson and Ingvar Åberg
}

IEEE Journal of Photovoltaics, 2016, Online

DOI and link to the original publication

DOI: $10.1109 / \mathrm{JPHOTOV} .2016 .2604564$

Open-Access full-text available at the Journal's site at this link:

http://ieeexplore.ieee.org/document/7575723/

C 2016 IEEE. Personal use of this material is permitted. Permission from IEEE must be obtained for all other users, including reprinting/republishing this material for advertising or promotional purposes, creating new collective works for resale or redistribution to servers or lists, or reuse of any copyrighted components of this work in other works. 


\title{
Performance of GaAs Nanowire Array Solar Cells for Varying Incidence Angles
}

\author{
Omid Madani Ghahfarokhi, Nicklas Anttu, Lars Samuelson and Ingvar Åberg \\ The authors are with Sol Voltaics AB, Lund 22363, Sweden (e-mail: omid.madani@solvoltaics.com; \\ nicklas.a@solvoltaics.com; lars.samuelson@ftf.lth.se; ingvar.aberg@solvoltaics.com;).
}

\begin{abstract}
Nanowire array solar cells show intrinsic light trapping and absorption enhancement properties due to the diffraction and optical resonances. Here, we report the effect of varying incidence angle on the performance of GaAs nanowire array solar cells. We provide experimental evidence that nanowire array solar cells are highly efficient at gathering diffuse or tilted incident light even at very high incidence angles; hence the performance of the nanowire solar cell is retained until these high incident light angles. Specifically, the measured efficiency at an incidence angle of $60^{\circ}$ is $95 \%$ of the efficiency at normal incidence. Moreover, our measurements show that a non-zero incidence angle is beneficial for wavelengths above $600 \mathrm{~nm}$, which results in an efficiency improvement by $0.5 \%$ absolute-points. This increase is so large that we even measure a small increase in absolute output power at $15^{\circ}$ tilt, thus more than fully compensating for the reduced incoming power over the cell with increasing tilt. We show how this gain arises from an enhanced absorption in the part of the nanowire with a high probability of charge extraction. Thus, nanowires show great promise to deliver high efficiency in practical non-tracking positioning conditions as well as under diffuse light illumination.
\end{abstract}

\section{Introduction}

III-V semiconductor materials offer excellent properties for various types of opto-electronic applications including photovoltaics. In particular, GaAs is of great interest for single junction solar cells due to its optimum band gap relative to the 1 sun air mass 1.5 global (AM1.5G) spectrum [1]. So far, the highest single junction solar cell efficiency under one sun illumination was achieved with a GaAs cell $[2,3]$. The most conventional approach to fabricate GaAs planar structures is through metalorganic vapor phase epitaxy (MOVPE). However, the epitaxial growth of GaAs layers grown by MOVPE is expensive, due to the high cost of organometallic precursors, as well as the cost of the MOVPE reactor and the single crystal GaAs substrate. Moreover, the use of precursors is inefficient in MOVPE [4]. These limitations have until now hindered the use of GaAs for one sun solar cell modules.

Nanowire-based solar cells are promising candidates for future solar cell applications due to their advantageous properties such as improved light trapping and reduced material need [5-10]. For 
example, the recent demonstration of aerotaxy, a substrate-less continuous growth system based on vapor-liquid-solid (VLS) growth, could eliminate the need for a growth substrate while at the same time increasing growth rates by orders of magnitude [11]. Furthermore, the nanowire geometry allows the absorption of more than $90 \%$ of the incident photons with energy above the band gap when we choose appropriate geometrical parameters such as nanowires diameter, nanowire length and array pitch $[10,12,13]$. Such geometry optimization leads to excellent wavecoupling and subwavelength optical concentration in nanowires. As a result, the material consumption can be reduced by up to one order of magnitude while the concentrated light improves the open-circuit voltage $\left(\mathrm{V}_{\mathrm{OC}}\right)[5]$.

Recently, we demonstrated a record efficiency of $15.3 \%$ for GaAs nanowire-array based solar cells [9]. In this study, we address the effect of the angle of incidence on the performance of GaAs nanowire array solar cells. First, the nanowire geometry introduces absorption properties beyond the simple Beer-Lambert law of exponential intensity decay with depth into the cell, in strong contrast to a conventional planar cell. Second, the probability to extract the photogenerated charge carriers depends on the axial position along the nanowires. Third, with a tilted, non-normal incidence angle, we tend to increase the photogeneration rate in regions of high extraction probability. As an end result, nanowire array solar cells are highly efficient at gathering diffuse or tilted incident light even at very high angles of incidence; therefore the efficiency of the nanowire array solar cell stays high until high incidence angles (up to $60^{\circ}$ incidence). This demonstrates the potential of a fixed-installation nanowire array solar cell for delivering large power output during the cycle of illumination from sunrise to sunset, as well as during diffuse illumination conditions.

\section{Experimental details and modeling method}

We have used GaAs (111)B substrates decorated with a square grid pattern of Au disks (500 nm pitch) where the Au disks were used as catalyst material for GaAs nanowire growth. The nanowire growth was performed using MOVPE at a growth temperature of $400^{\circ} \mathrm{C}$. Trimethylgallium (TMGa) and arsine $\left(\mathrm{AsH}_{3}\right)$ were used as precursors for the GaAs nanowire growth. Diethyl zinc (DEZn) and tetraethyl tin (TESn) were used as dopant precursors to fabricate, respectively, $\mathrm{p}$ - and $\mathrm{n}$-type doped segments in a $p-n$ axial geometry. Following the $p-n$ axial nanowire growth, the surfaces of the nanowires were passivated by growing a radial AIGaAs shell (20-40 nm shell thickness) at a temperature of $715^{\circ} \mathrm{C}$. To fabricate the solar cells, a partially planarized surface was then formed by depositing silicon oxide and spin-coating CYCLOTENE. To form the front contact, these layers were etched back to reveal the top of the nanowires, the Au catalyst particles were removed and finally an approximately $100 \mathrm{~nm}$ thick indium tin oxide (ITO) top contact layer was deposited. Subsequently, on the substrate, multiple solar cells of $1 \mathrm{~mm}^{2}$ in area were defined by patterning the ITO. The detailed description of the fabrication process is reported in Ref. [9]. 
A Newport ORIEL IQE 200 setup was used for external quantum efficiency (EQE) measurements. The I-V characteristics of the solar cells were measured at room temperature under the 1 sun AM1.5G spectrum of intensity $W_{\text {inc }}=1000 \mathrm{~W} / \mathrm{m}^{2}$, using a Newport Sun simulator. The power conversion efficiency $(\eta)$ was then obtained from $\eta=\frac{P_{\text {out }}}{P_{\text {in }}}$, where $P_{\text {out }}=I_{\mathrm{SC}} * F F * V_{\mathrm{OC}}$ is the output power and $P_{\text {in }}$ is the incident power. For a tilted cell of area $A_{\text {cell }}, P_{\text {in }}=W_{\text {inc }}{ }^{*} A_{\text {proj, }}$, with $A_{\text {proj }}$ $=\cos (\theta) * A_{\text {cell }}$ where $\theta$ is the angle between the solar cell and the horizon as shown in Fig. 1a (note that this geometry configuration is equivalent to tilting the incidence angle by $\theta$ for a fixed cell, as shown in Fig. 1b). Thus, the efficiency $\eta=I_{\mathrm{sc}} / A_{\text {proj }} * F F * V_{\mathrm{oc}} / W_{\text {inc }}$ is proportional to $I_{\mathrm{SC}} / A_{\text {proj. }}$. Conventionally, for an untilted cell with $\theta=0$, the short circuit current $\left(J_{\mathrm{SC}}\right)$ is defined as $I_{\text {sc }}$ divided by the cell area $A_{\text {cell. }}$. However, here, in the analysis of the performance of the tilted cells, we concentrate on this more relevant quantity $I_{s c} / A_{\text {proj. }}$.

To aid in the analysis of the experimental results, optics simulation of the absorption of light was performed with the scattering matrix method [14]. In this method, the diffraction of light is modeled with Maxwell's equations. For the simulation, GaAs nanowire arrays were defined with the length, diameter, and pitch of 2800, 165, and $500 \mathrm{~nm}$, respectively. $100 \mathrm{~nm}$ thick ITO layer and a $40 \mathrm{~nm}$ thick AlGaAs shell were introduced in the model. The incident media is air with refractive index $n_{\text {incident }}=1$, and $n_{\text {filler }}=1.5$ is set for the filler between the nanowires. In this modeling, the light is incident as a plane-wave from a direction given by the angle $\theta$ with respect to normal incidence as shown in Fig. 1b. To model the experimental case of unpolarized light, we averaged the results for transverse electric (TE) and transverse magnetic (TM) polarized light. Figure $1 \mathrm{~b}$ shows a schematic illustration of the defined structure for the simulation and the illumination direction.

The optical properties of the constituent materials are described with their respective wavelength dependent refractive index. References [15], [16] and [17] and are used for the refractive index of GaAs, AlGaAs and ITO, respectively. We modeled the AIGaAs shell as Al0.6Ga0.4As. To simplify the modeling, we set the imaginary part of the refractive index of this AlGaAs to zero. With this approximation, we neglect a possible absorption of photons in the shell. Such absorption could have some effect on the total absorption in the high energy $(\lambda<500 \mathrm{~nm})$ region where the AlGaAs shows strong direct optical transitions. However, for $\lambda>500 \mathrm{~nm}$, we expect negligible absorption in the AlGaAs shell due to weak, indirect optical transitions. In contrast, noticeable absorption in the ITO can in principle show up throughout the whole wavelength range where the GaAs nanowire core absorbs, that is, for $\lambda<870 \mathrm{~nm}$. Therefore, we included into the modeling the absorption losses in the ITO layer by using the complex-valued refractive index of the ITO. 


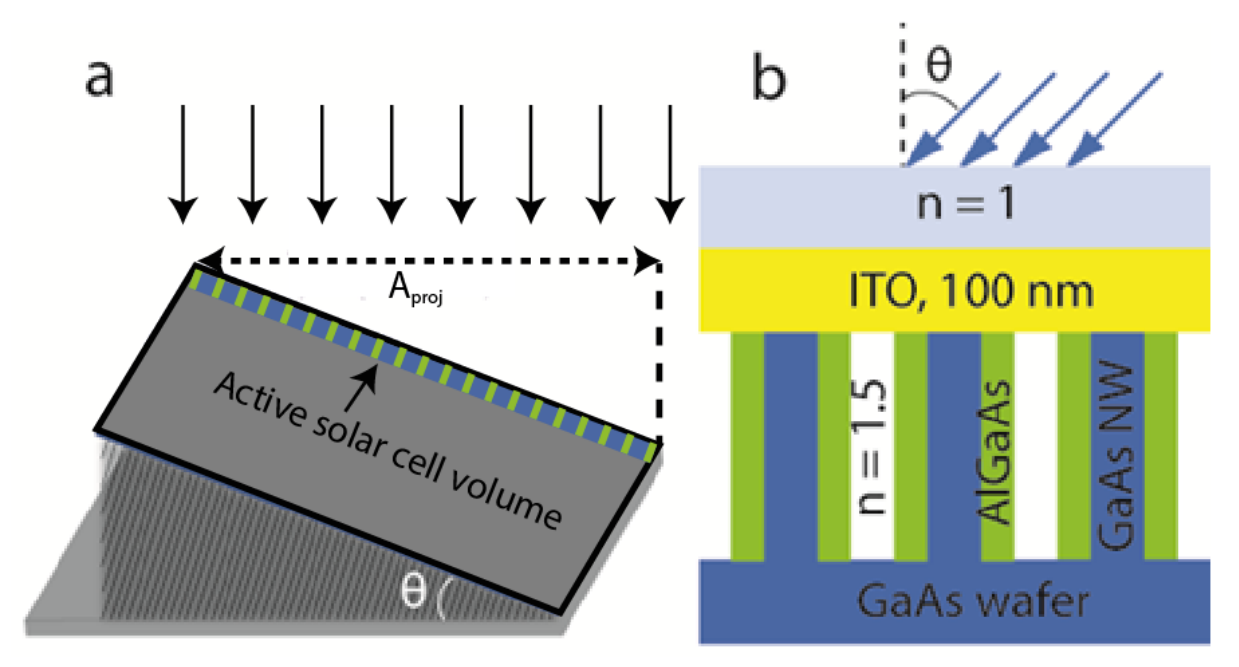

Fig. 1- (a) Schematic illustration of a solar cell measured under tilt angle of $\theta$. (b) Schematic illustration of the defined structure for the simulation. For the simulation, instead of tilting the solar cell, the incoming light is tilted, that is, light is incident at an angle $\theta$.

\section{Measured dependence of performance on tilt angle}

We have measured the electrical properties of three GaAs nanowire solar cells fabricated on two different GaAs substrates for varying tilt angle with respect to the incident light, including the case of normally incident light, that is, for $\theta=0$.

First, we concentrate on current, as measured by $I_{s c} / A_{\text {proj }}$ (Fig. 2). We find an increase of $I_{s c} / A_{\text {proj }}$ of $\approx 0.8 \mathrm{~mA} / \mathrm{cm}^{2}$ (the average of the three samples) at $15^{\circ}$ tilt angle relative to no tilt. The $/ \mathrm{sc} / A_{\text {proj }}$ increase is $\approx 0.6$ and $0.3 \mathrm{~mA} / \mathrm{cm}^{2}$ at $30^{\circ}$ and $45^{\circ}$ tilt angle, respectively. Interestingly, even at a tilt angle of $60^{\circ}, 95 \%$ of the $I_{s c} / A_{\text {proj }}$ is retained compared to the normal incident condition providing experimental evidence that nanowire array solar cells are highly efficient at gathering diffuse or tilted incident light even at very high angles of incidence. Note that the absolute current output Isc of the solar cell decreases with $\theta$ (inset in Figure 3 ), as expected from the $\cos (\theta)$ dependence in the projected illumination area $A_{\text {proj }}=A_{\text {cell }} * \cos (\theta)$. 


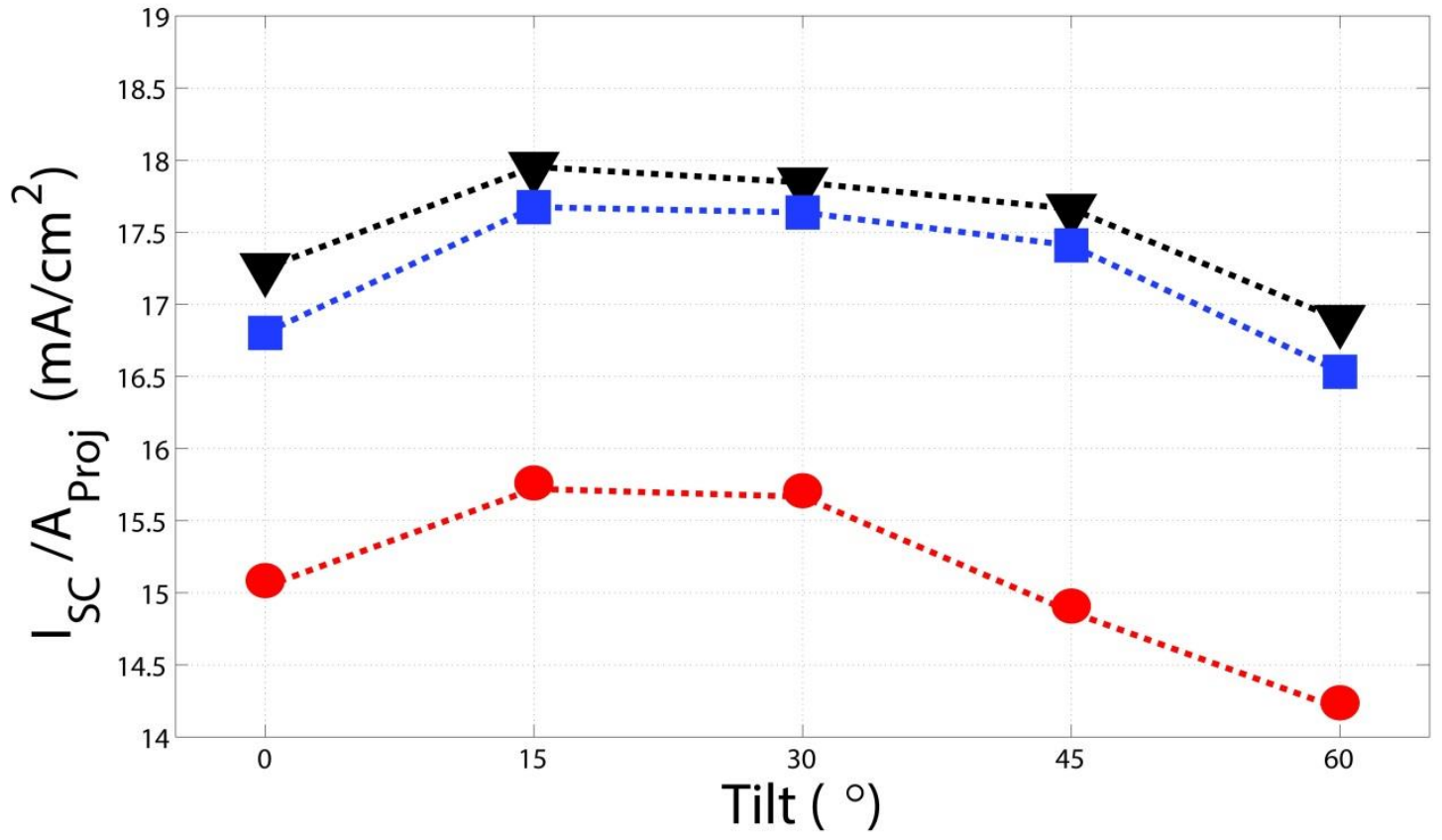

Fig. 2- The dependence of $/ s c / A_{\text {proj }}$ on the tilt angle $\theta$ for three different GaAs nanowire solar cells.

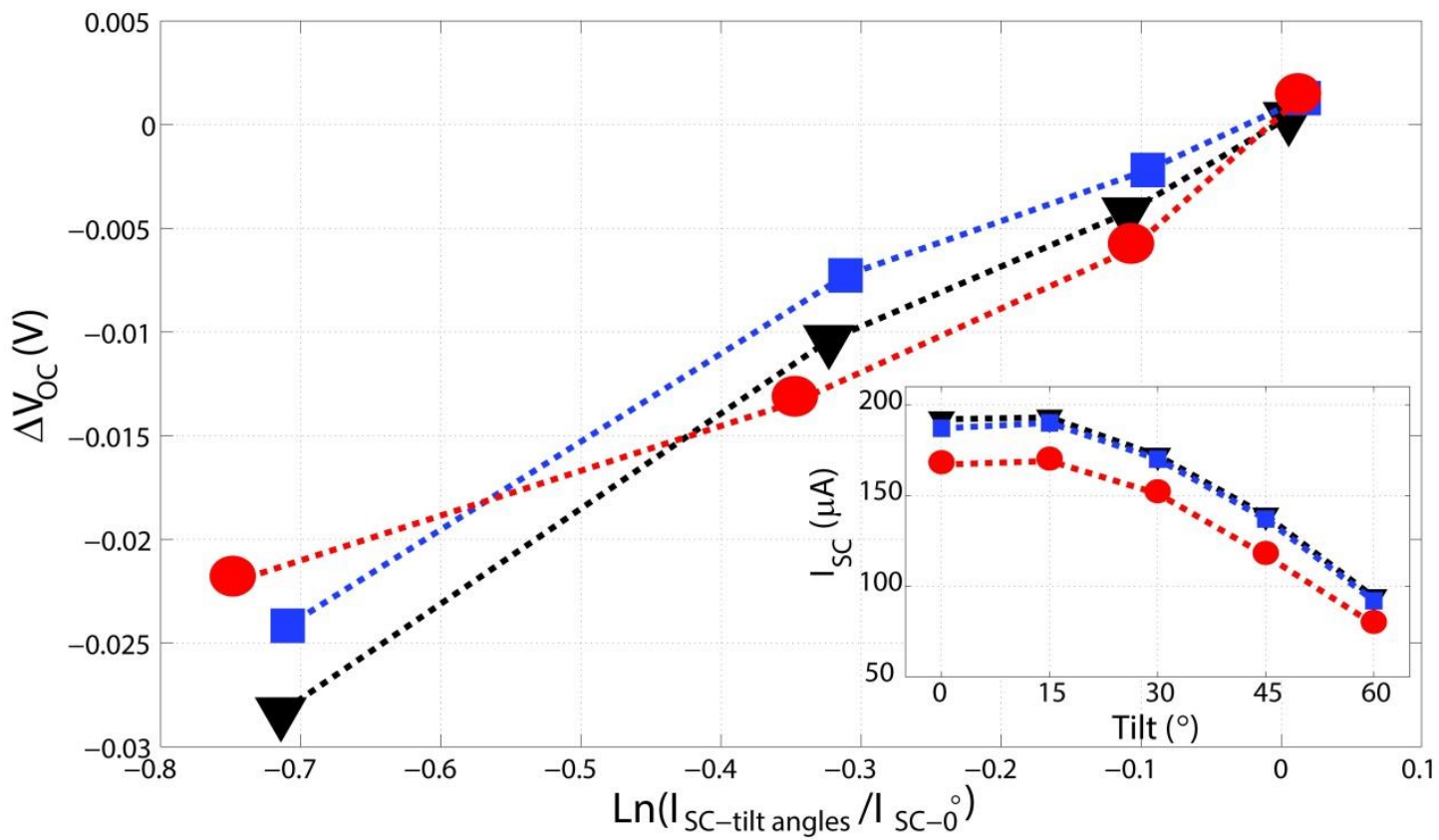

Fig. 3- The variation in $V_{\mathrm{OC}}$ (compared to the $0^{\circ}$ tilt angle) with respect to the variation in measured current. The data points represent $15,30,45$ and $60^{\circ}$ tilt angle from right to left, respectively. The inset shows the variation in $I_{s c}$ with respect to the tilt angle from $0-60^{\circ}$ tilt. 
Next, we investigate the dependence of the voltage performance of the solar cell (Fig. 3). We find a linear dependence of the open-circuit voltage $\left(V_{\mathrm{OC}}\right)$ as a function of $\ln \left(I_{\mathrm{sc}}\right)$. Note that such a linear dependence indicates that the solar cell is characterized by an ideality factor $n$ for operation around the open-circuit condition. In such a regime, the IV-curve is given by $I=I_{\mathrm{SC}}-$ $I_{0} \exp (q V /(n k T))$, from which the $V_{\mathrm{OC}}$ is given by $V_{\mathrm{OC}}=\frac{n k T}{q} \ln \left(\frac{I_{\mathrm{SC}}}{I_{0}}-1\right) \approx \frac{n k T}{q} \ln \left(\frac{I_{\mathrm{SC}}}{I_{0}}\right)$ where $n, k$, $T, q$, and $I_{0}$ are the ideality factor, the Boltzmann constant, the temperature of the cell, the elementary charge, and the dark saturation current, respectively. Apart from $I_{\mathrm{Sc}}$, none of the other parameters are expected to depend on the illumination angle. Hence, we can define the variation in $V_{\text {oc }}$ with respect to the zero tilt case as

$\Delta V_{\mathrm{OC}}=V_{\mathrm{OC}-\mathrm{tilt}}-V_{\mathrm{OC}-0^{0}}=\frac{n k T}{q} \ln \left(\frac{I_{S C-t i l t}}{I_{S C-0^{0}}}\right)=B * \ln \left(\frac{I_{S C-t i l t}}{I_{S C-0^{0}}}\right)$,

where $B$ is a constant. The measured $\Delta V_{\mathrm{OC}}$ agrees well with this description, as seen from the linear increase of $\Delta V_{\mathrm{OC}}$ with $\ln \left(\frac{I_{S C-t i l t}}{I_{S C-0^{0}}}\right)$ in Fig. 3. From linear fitting of the results in Fig. 3 to Eq. (1), we find an ideality factor of $n \approx 1.54$. When we instead turn to study the $V_{\text {oc }}$ as a function of incidence angle (Fig. 4a), we find for all three samples a noticeable drop at $60^{\circ}$ tilt angle. From Eq. (1), we attribute this drop to the lower absolute current level Isc (inset in Fig. 3), due to the decreased, projected illumination area $A_{\text {proj }}$ as discussed above. In contrast, the measured fill factor $(F F)$ is nearly independent or possibly even slightly increasing with tilt angle (Fig. 4b). Such an increase in FF may be linked to the lower absolute current level, which could decrease resistive losses in the cell.

Finally, when turning to study the efficiency of the cells (Fig. 4c), we find an increase by about $0.5 \%$ point in efficiency for all the three samples for $\theta=15^{\circ}$ as compared to $\theta=0^{\circ}$. It should be mentioned that the measured NW solar cell samples not only have higher efficiency (at $15^{\circ}$ tilt angle) but also deliver higher actual output power (see Fig. 4d). Thus, the increase in efficiency of a NW solar cell at the optimum tilt angle can more than fully compensate the loss in input power (due to the $\cos (\theta)$ factor from the tilting of the cell) and deliver higher output power (as compared to the $0^{\circ}$ tilt).

It should be noted that for the case of a conventional crystalline silicon cell, or even for a black silicon solar cell, neither higher efficiency nor higher power is obtained for any tilt angle compared to normal incident light [18]. This unique property of nanowire array solar cells shows their potential for diffuse light illumination, as well as for practical fixed-installation conditions where the illumination angle varies over the course of the day. 

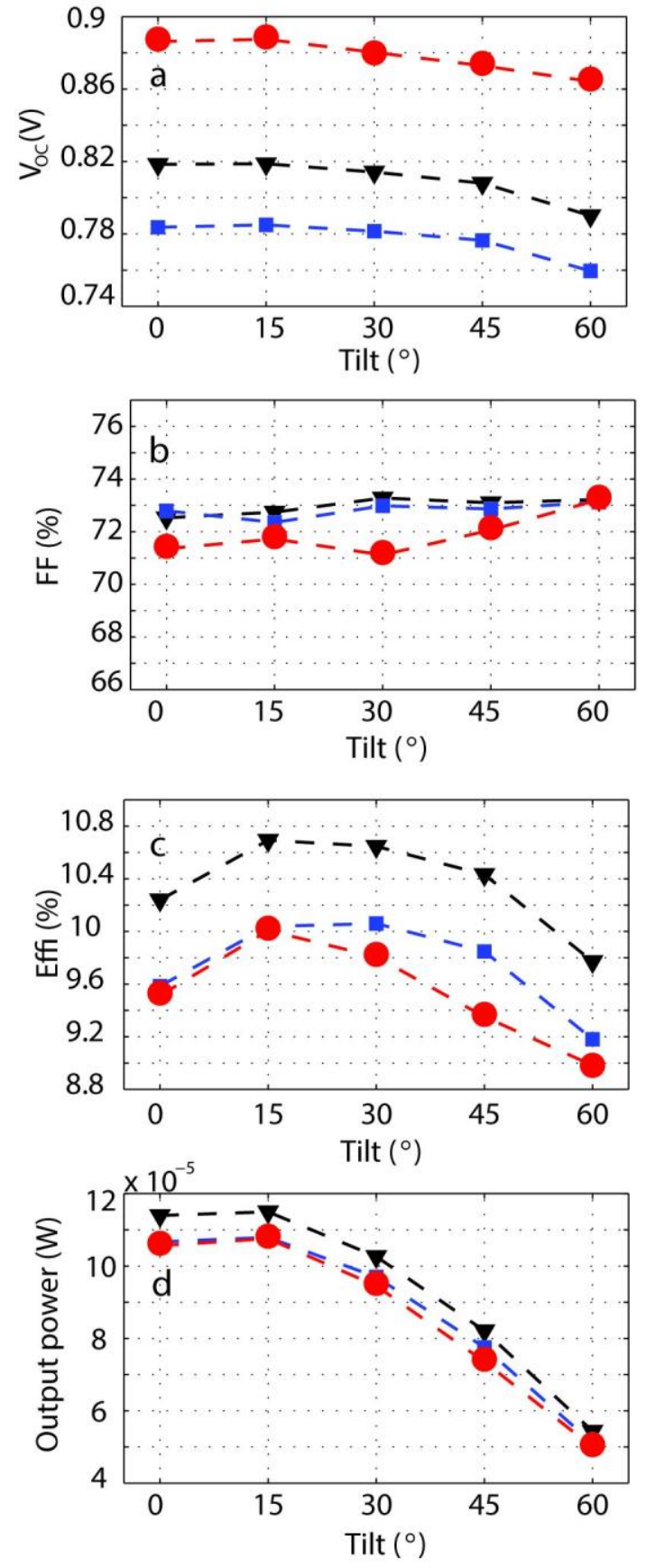

Fig. 4- The variation of (a) $V_{o c}$, (b) FF, (c) efficiency $\eta$ and (d) output power ( $\left.P_{\text {out }}=I_{s c} * F F^{*} V_{o c}\right)$ at one sun with respect to the tilt angle from $0-60^{\circ}$ tilt. The three data sets in each graph correspond to the three measured cells. 


\section{Analysis of current generation performance}

Above, we saw that it is the strong photogeneration and charge-carrier extraction ability (as characterized by $I_{s c} / A_{\text {proj}}$ ) of the nanowire array solar cell at high tilted incidence that drives the increase in efficiency and even produces a slight increase in output power for a tilt angle of $15^{\circ}$. Here, to get a deeper understanding about the $I_{s c} / A_{\text {proj }}$ behavior, we have measured the EQE of the GaAs solar cell whose IV charactertistics are marked with triangles in Figs. 2-4. We performed the EQE analysis for varying tilt angle in steps of $15^{\circ}$ up to $\theta=60^{\circ}$ (Fig. 5). For $0^{\circ}$ tilt, we find a peak in the EQE at approximately $\lambda=570 \mathrm{~nm}$ in wavelength. The peak position shifts to $680 \mathrm{~nm}$ wavelength for all considered non-zero tilt angles. Moreover, for non-zero tilt, we identify two regions with different dependence compared to $0^{\circ}$ tilt. First, relatively lower EQE signals are measured for wavelengths between $\approx 520-600 \mathrm{~nm}$. Based on simulation results (Fig. $6 \mathrm{~b}$ ), this could be attributed for example to the slightly increased reflection at these wavelengths for the case of non-zero tilt. Second, in the region between $610 \mathrm{~nm}$ and $850 \mathrm{~nm}$, the EQE is improved for tilt angles in the range of $15-45^{\circ}$. Hence, we conclude that the higher measured $I s c / A_{\text {proj }}$ at non-zero tilt angles of $15-45^{\circ}$ in Fig. 2 is due to this increase in EQE in the long-wavelength part of the incident spectrum.

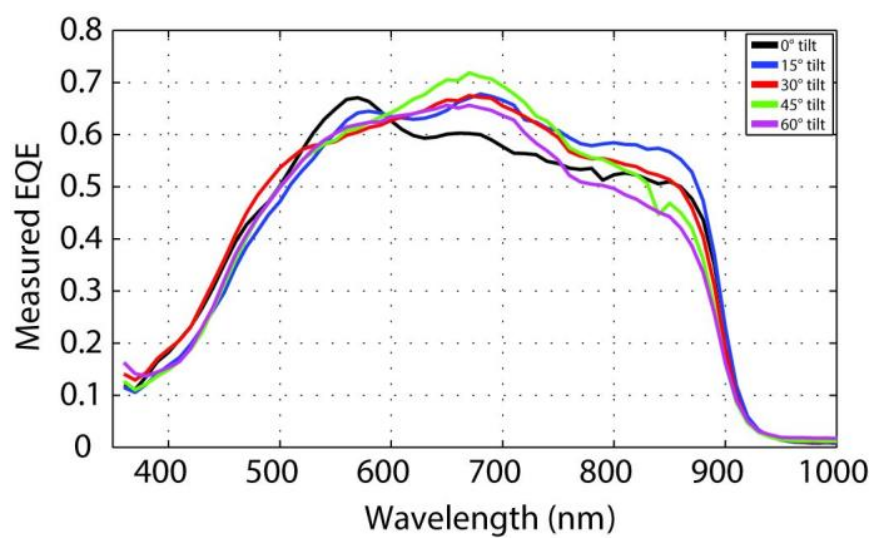

Fig. 5- The measured external quantum efficiency (EQE) for a GaAs nanowire solar cell with $500 \mathrm{~nm}$ pitch measured at $0^{\circ}$ to $60^{\circ}$ tilt angle. Higher EQE signal is measured in the red part of the spectrum (between $\approx 600-850 \mathrm{~nm}$ wavelengths) for almost all the tilt angles, as compared to the case of zero tilt. 

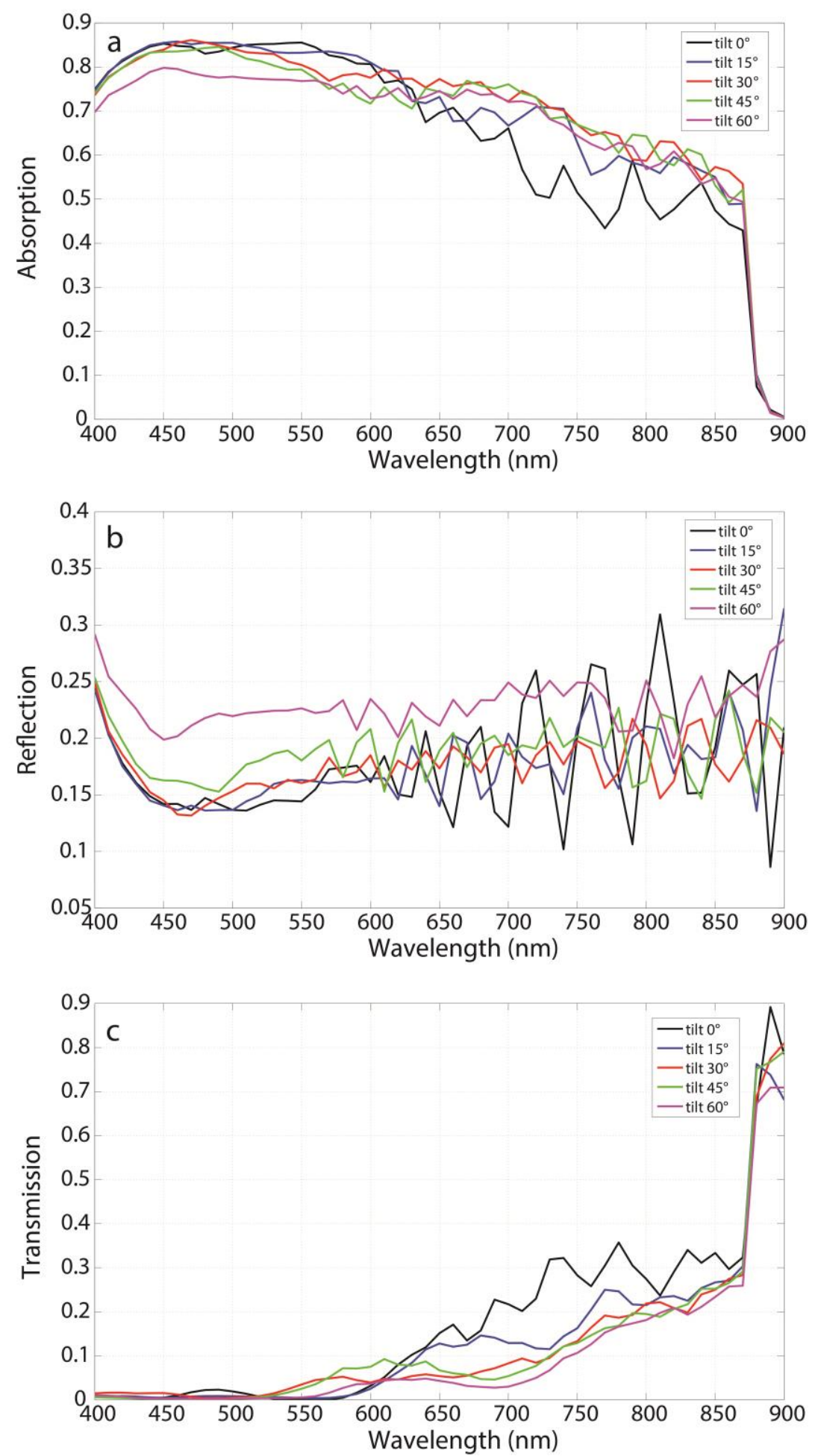

Fig. 6- The simulated (a) absorption, (b) reflection and (c) transmission spectra of a GaAs nanowire array (nanowire length: $2800 \mathrm{~nm}$, nanowire diameter: $160 \mathrm{~nm}$, array pitch: $500 \mathrm{~nm}, \mathrm{n}_{\text {incident }}:$ 1, $\mathrm{n}_{\text {filler: }}: 1.5$ and $\mathrm{n}_{\text {substrate: }}$ GaAs) for different tilt angles. Note that all non-zero tilt-angles lead to higher absorption than 0 tilt angle for wavelengths longer than $650 \mathrm{~nm}$. 
To understand this increase in the EQE at long wavelengths, we have simulated the optical response of the nanowire array with the scattering matrix method for varying incidence angle (see Section 2 above for details). We present the fraction of light absorbed in the nanowires (Fig. $6 a)$, the reflection back to the top air side (Fig. 6b) and the transmission into the underlying substrate (Fig. 6c).

The simulated absorption is enhanced for tilted incident light at wavelengths above $650 \mathrm{~nm}$ (Fig. $6 a)$, in line with the corresponding increase in the measured EQE of Fig. 5. The average reflection (between $600-850 \mathrm{~nm}$ in wavelength) increases only slightly, from $18 \%$ to $22 \%$, as the incidence angle is tilted from $0^{\circ}$ to $60^{\circ}$. In contrast, the average transmission (between $600-850 \mathrm{~nm}$ in wavelength) shows a much stronger dependence on the incidence angle and drops from $22 \%$ at $\theta=0^{\circ}$ to $15 \%$ at $\theta=15^{\circ}$, and to $12 \%$ for $\theta=30^{\circ}$ and $45^{\circ}$. Hence, we deduce that by tilting the NW array, the reflection does not vary much whereas the transmission reduces. Thus, the increased absorption in the nanowire array decreases the amount of light that can transmit to the substrate.

To understand the absorption behavior even better, we have studied how the absorption is distributed along the axial direction of the nanowire, that is, with depth into the nanowire array. Figure 7 shows such absorption depth profiles for $550 \mathrm{~nm}$ and $800 \mathrm{~nm}$ wavelength for $0^{\circ}$ and $30^{\circ}$ tilt (the corresponding cumulative absorption is shown in the inset). For comparison, we also show the absorption for a $2.8 \mu \mathrm{m}$ thick planar GaAs layer, which follows the Beer-Lambert law of exponential decay with depth into the layer. In contrast, the nanowire array shows a more complicated dependence with multiple peaks of varying periodicity. 


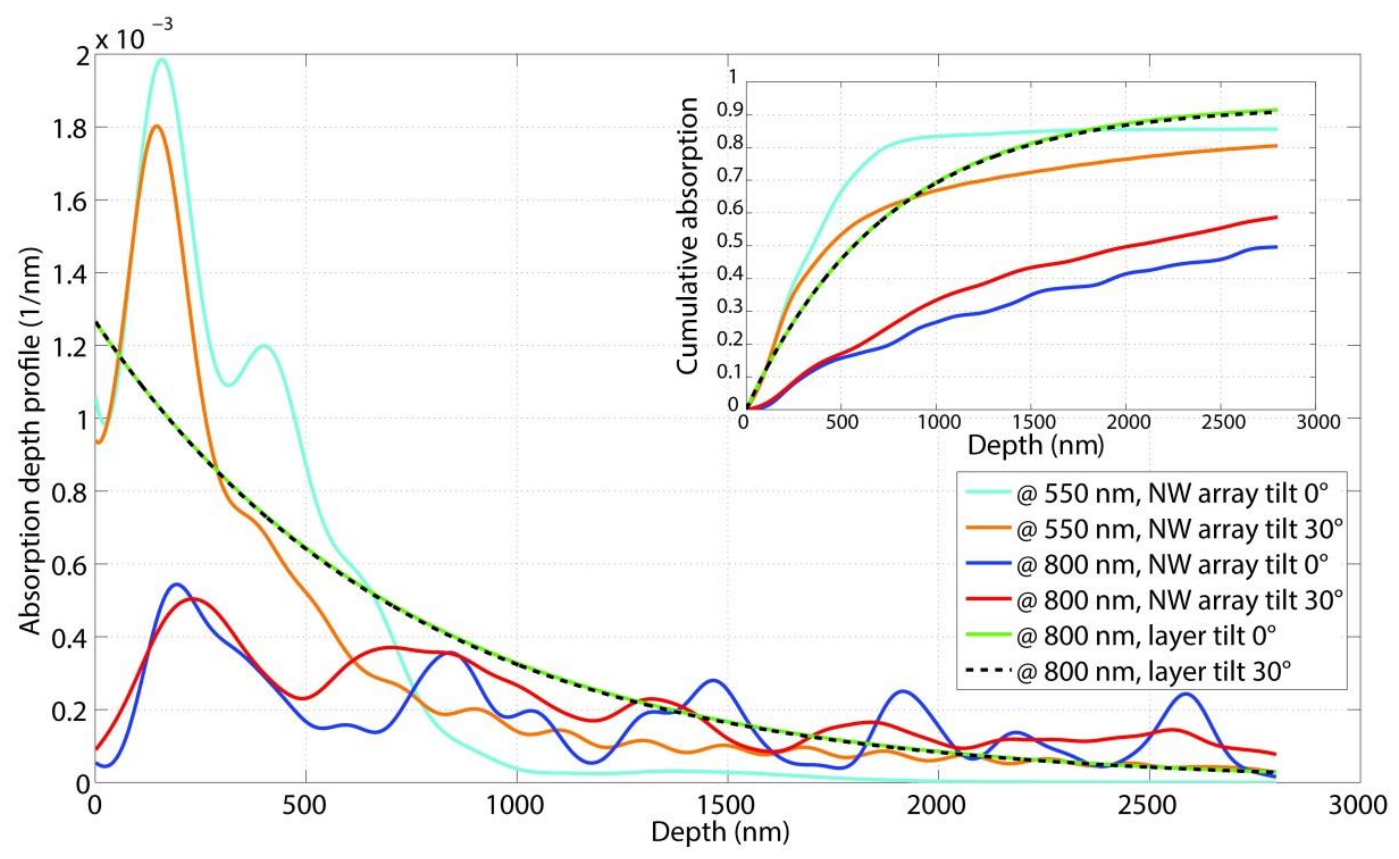

Fig. 7- Absorption depth profile for 550 and $800 \mathrm{~nm}$ wavelength for a GaAs nanowire array and for $800 \mathrm{~nm}$ wavelength for a GaAs layer at $0^{\circ}$ and $30^{\circ}$ tilted angle. The inset shows the corresponding cumulative absorption of the GaAs NW array and the layer.

Next, we relate the absorption depth profiles to the AM1.5G solar spectrum $I_{\text {AM1.5 }}(\lambda)$ used in the experiments. Note that we can translate the absorption depth profile $F(\lambda, z)$ into a photogeneration depth profile as $G_{z}(\mathrm{z})=\int \mathrm{d} \lambda I_{\mathrm{AM} 1.5}(\lambda) F(z, \lambda) /(2 \pi \hbar c / \lambda)$ [13]. We show such photogeneration depth profiles for $0^{\circ}$ and $30^{\circ}$ incidence angle in Fig. 8 (with the corresponding cumulative photogeneration profile in the inset). In the first $300 \mathrm{~nm}$ of depth along the nanowire length axis, the absorption is similar between the two cases. However, between $300 \mathrm{~nm}$ and 1400 $\mathrm{nm}$, the cumulative photogeneration profiles for $0^{\circ}$ and $30^{\circ}$ tilt diverge (inset in Fig. 8), with a higher absorption for the tilted incidence. In the second half of the nanowire, from a depth of $1400 \mathrm{~nm}$, the absorption is similar for both tilt angles, and the two cumulative photogeneration profiles are parallel. Quantitatively, the cumulative absorption is approximately $5 \%$ higher over the whole nanowire for the $30^{\circ}$ tilt. Thus, the simulated absorption is higher in a $30^{\circ}$ tilted nanowire array PV cell, qualitatively consistent with the measured increase in $I s \mathrm{~s} / A_{\text {proj }}$ and the increase in efficiency. However, for a more complete analysis, not only the absorption but also the likelihood of collecting the photogenerated carriers should be considered. 


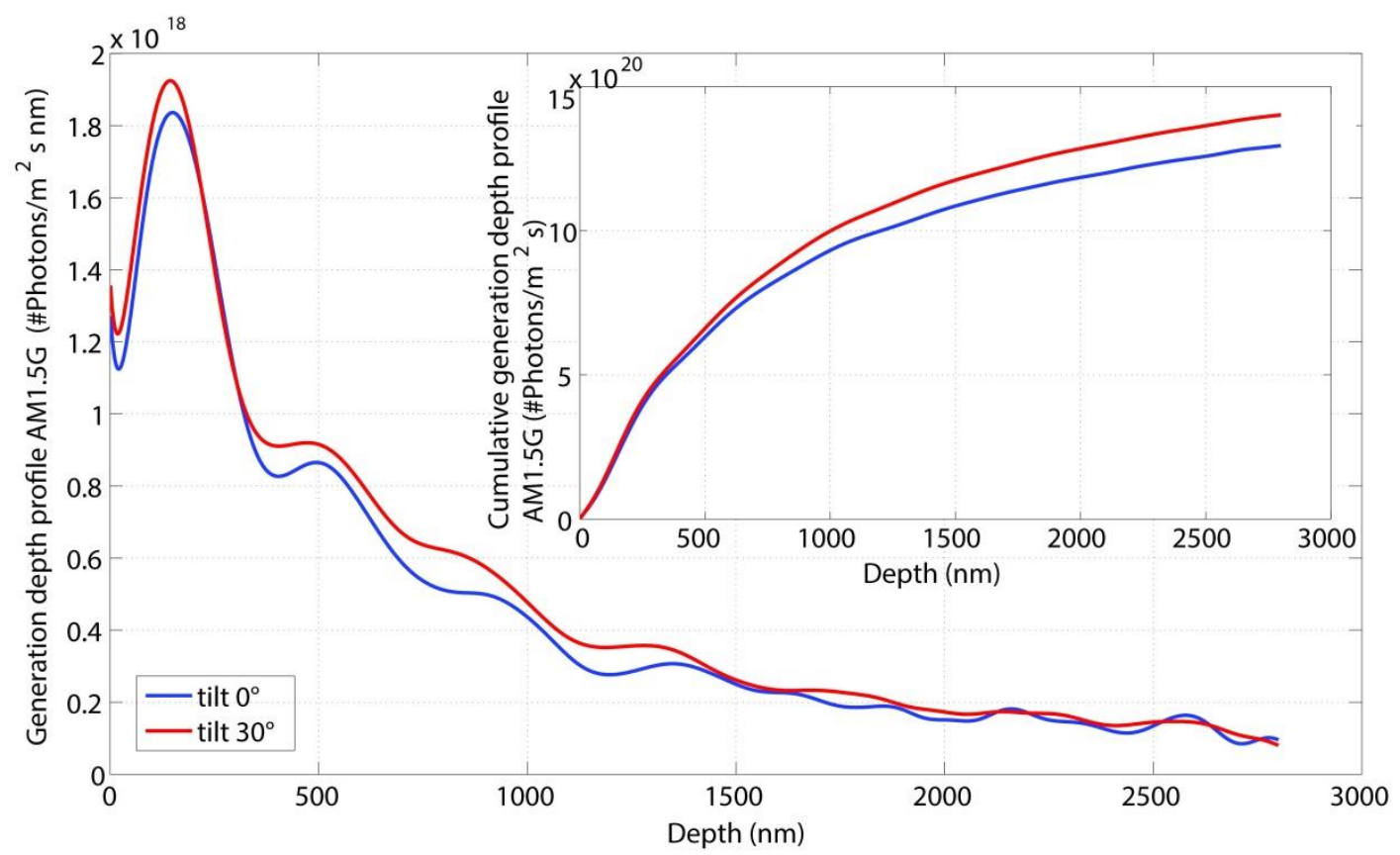

Fig. 8-Calculated photogeneration depth profile of GaAs NW array under AM1.5G illumination for $0^{\circ}$ and $30^{\circ}$ tilt. The inset shows the cumulative photogeneration for AM1.5G illumination. Between a depth of approximately $300 \mathrm{~nm}$ and $1400 \mathrm{~nm}$, the AM1.5G absorption is higher for the tilted case, causing the cumulative absorption curves to diverge.

For the current generation performance, in addition to the photogeneration rate, the probability to extract the photogenerated charges plays a major role. To assess the internal charge-carrier collection efficiency along the length of the nanowire, the electron beam-induced current (EBIC) response of a single nanowire was also measured, after the fabrication step of the AIGaAs shell, using the substrate as the back contact and the Au catalyst particle as the top contact. Such EBIC response is expected to be proportional to the probability to extract photogenerated charges. The EBIC response and the corresponding SEM micrograph of the measured nanowire are shown in Figs. 9a and 9b, respectively. The relative EBIC profile at the center of the cross-section of the wire, as a function of the length along the wire axis, is shown in Fig. 9c. The profile consists of three regions with considerably different behavior: 1 ) $n$-type emitter region (of approximately $200 \mathrm{~nm}$ in length) which shows a sharply decreasing signal toward the Au tip of the nanowire, which we attribute to recombination of photogenerated holes (the minority carriers) in the Au particle or the contact [9], 2) a nearly flat and high response region (until $1500 \mathrm{~nm}$ depth) which we attribute to either charge separation by an electric field in a depletion region or diffusion in a quasi-neutral absorber characterized by a long diffusion length, and 3) a last region (deeper than $\sim 1500 \mathrm{~nm}$ depth) near the base of the wire where the response decreases toward the substrate. In this last region, we attribute the decrease in the response to a decreasing probability for 
electrons (the minority carriers) to diffuse to the depletion region with increasing distance from the depletion region.

To obtain the probability for charge extraction from this EBIC response, we assumed that the extraction probability is zero at the edge of the Au particle and $100 \%$ at the maximum point in the plateau region. After this, we convoluted this extraction profile with the simulated depth generation profile to yield a modeled EQE. We show the measured and modeled EQE for normal and $30^{\circ}$ tilted incidence in Fig. 9d. In agreement with measured data, a higher modeled EQE is found for $650-850 \mathrm{~nm}$ wavelengths for the $30^{\circ}$ tilt compared to $0^{\circ}$ tilt. By considering the measured EBIC profile (Fig. 9c) and the calculated absorption depth profiles (Fig. 7a), it can be concluded that by tilting the nanowire array solar cell, the photogeneration increases in the region that shows a higher probability for extracting photogenerated carriers. This overlap of the regions for enhanced photogeneration and high extraction probability results in the observed increase in $/ \mathrm{sc} / A_{\text {proj. }}$

The discrepancy between the measured and modeled EQE for wavelengths smaller than $550 \mathrm{~nm}$, where the measured EQE drops faster than the modeled EQE, is attributed to absorption in the AlGaAs passivation layer. As discussed in Section 2, such absorption was not included in the modeling for simplicity. We believe that in the experiments, a large fraction of the photogenerated charges in the AIGaAs shell recombine (due to lack of proper surface passivation of the shell), before transfer into the GaAs core where they could contribute to the current, leading to a drop in the measured EQE. Thus, increasing the Al concentration in the AIGaAs layer should further increase the Isc of the nanowire array solar cell. 

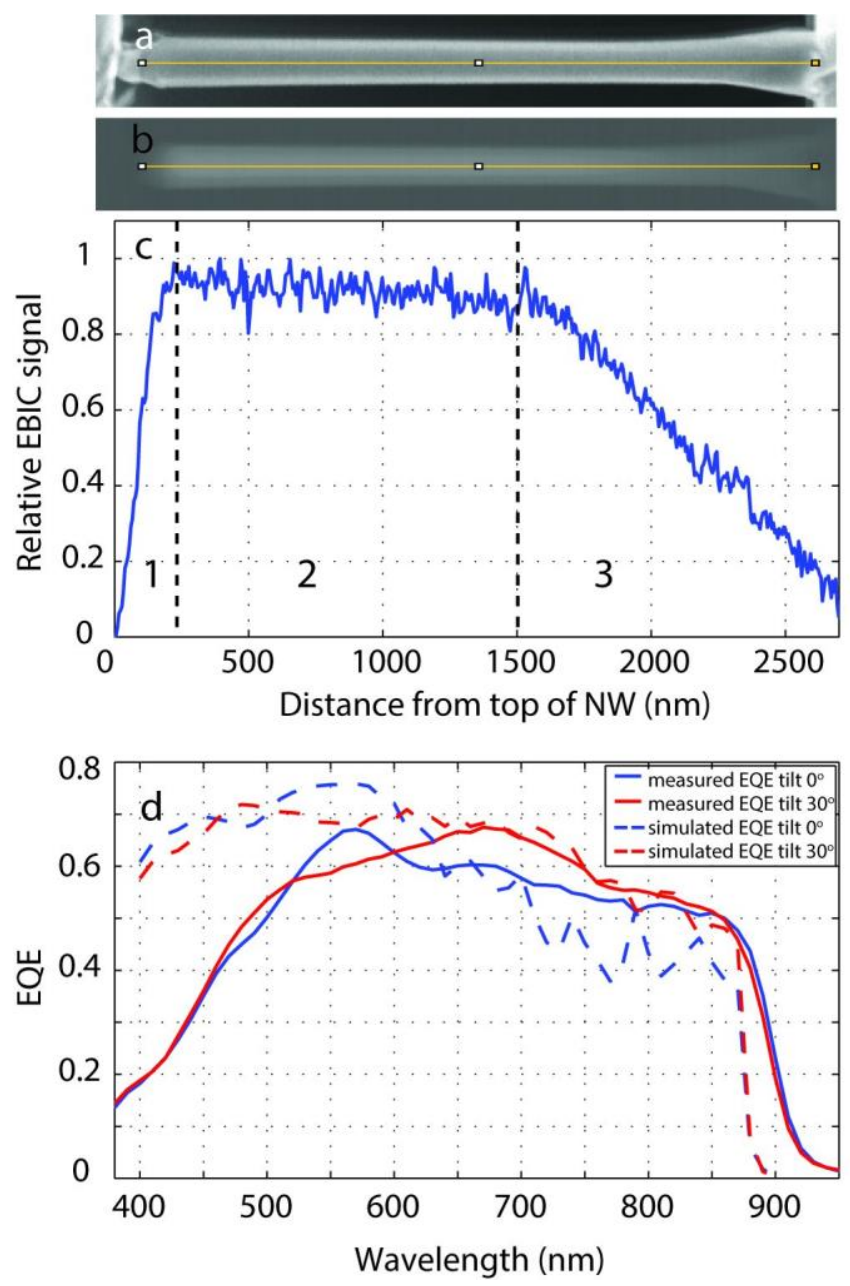

Fig. 9- (a) High magnification SEM and (b) EBIC measurement of a nanowire grown similarly as the nanowires in the measured nanowire-array solar cells. The GaAs substrate and the Au particle are seen on the right and left edges of the images, respectively. (c) The measured EBIC profile along the center of the nanowire. (d) The measured (line) and simulated (dashed line) EQE signal for the normal incident and $30^{\circ}$ tilted angle. The simulated EQE is calculated by multiplying the simulated wavelength-resolved photogeneration depth profile with the extraction probability profile (obtained from the EBIC profile shown in (c)).

\section{Conclusion}

In summary, we have investigated the effect of tilted incidence on the electrical performance of GaAs nanowire-array solar cells. Our measurements show that by tilting the nanowire array solar cells, stronger absorption can take place within the nanowires as compared to with normal incident light. Our modeling indicates on average a 5\% higher absorption under AM1.5G for $30^{\circ}$ tilt compared to at normal incidence. For the nanowires in this study, this additional absorption occurs in a region of high probability to extract the photogenerated carriers, as shown by EBIC measurements on individual nanowires. As an end result, the efficiency increases by 
approximately $1 \%$ (relative) for $30^{\circ}$ tilt compared to normal incidence. This enhanced absorption can, for small tilt angle, more than compensate for the decrease in the incoming power over the cell with increasing tilt. Indeed, we measure a small increase in actual power output when increasing the tilt to $15^{\circ}$. Furthermore, the efficiency of the nanowire arrays solar cell at $60^{\circ}$ tilt is still $95 \%$ of the efficiency at normal incidence. The results indicate the prospect of nanowire array solar cells (compared to cells using planar absorber layers, even if textured) for delivering higher output power and consequently cheaper electricity generation.

\section{ACKNOWLEDGMENT}

This project has received funding from the European Union's Horizon 2020 research and innovation program under grant agreements No. 696519 and 641023. It has also been funded by the Swedish Energy Agency.

\section{References}

1. Shockley, W. and H.J. Queisser, Detailed Balance Limit of Efficiency of $p$-n Junction Solar Cells. Journal of Applied Physics, 1961. 32(3): p. 510-519.

2. Kayes, B.M., et al. 27.6\% Conversion efficiency, a new record for single-junction solar cells under 1 sun illumination. in Photovoltaic Specialists Conference (PVSC), 2011 37th IEEE. 2011.

3. Green, M.A., et al., Solar cell efficiency tables (version 47). Progress in Photovoltaics: Research and Applications, 2016. 24(1): p. 3-11.

4. Zheng, M., et al., III-Vs at scale: a PV manufacturing cost analysis of the thin film vapor-liquidsolid growth mode. Progress in Photovoltaics: Research and Applications, 2016: p. n/a-n/a.

5. Wallentin, J., et al., InP nanowire array solar cells achieving $13.8 \%$ efficiency by exceeding the ray optics limit. Science, 2013. 339(6123): p. 1057-60.

6. Krogstrup, P., et al., Single-nanowire solar cells beyond the Shockley-Queisser limit. Nature Photonics, 2013. 7(4): p. 306-310.

7. Cui, Y., et al., Efficiency Enhancement of InP Nanowire Solar Cells by Surface Cleaning. Nano Letters, 2013. 13(9): p. 4113-4117.

8. Mariani, G., et al., GaAs nanopillar-array solar cells employing in situ surface passivation. Nat Commun, 2013. 4: p. 1497.

9. Aberg, I., et al., A GaAs Nanowire Array Solar Cell With 15.3\&\#x0025; Efficiency at 1 Sun. Photovoltaics, IEEE Journal of, 2015. PP(99): p. 1-6.

10. Wu, Y., et al., Enhanced photovoltaic performance of an inclined nanowire array solar cell. Optics Express, 2015. 23(24): p. A1603-A1612.

11. Heurlin, M., et al., Continuous gas-phase synthesis of nanowires with tunable properties. Nature, 2012. 492(7427): p. 90-94.

12. Nicklas Anttu, A.A., Damir Asoli, Magnus Heurlin, Ingvar Åberg, Lars Samuelson, Magnus Borgström, Absorption of light in InP nanowire arrays. Nano Research, 2014. 7(6): p. 816-823.

13. Wallentin, J., et al., InP Nanowire Array Solar Cells Achieving 13.8\% Efficiency by Exceeding the Ray Optics Limit. Science, 2013. 339(6123): p. 1057-1060. 
14. Anttu, N. and H.Q. Xu, Scattering matrix method for optical excitation of surface plasmons in metal films with periodic arrays of subwavelength holes. Physical Review B, 2011. 83(16): p. 165431.

15. Aspnes, D.E. and A.A. Studna, Dielectric functions and optical parameters of Si, Ge, GaP, GaAs, GaSb, InP, InAs, and InSb from 1.5 to 6.0 eV. Physical Review B, 1983. 27(2): p. 985-1009.

16. Aspnes, D.E., et al., Optical properties of AlxGa1-x As. Journal of Applied Physics, 1986. 60(2): p. 754-767.

17. Holman, Z.C., et al., Infrared light management in high-efficiency silicon heterojunction and rearpassivated solar cells. Journal of Applied Physics, 2013. 113(1): p. 013107.

18. Savin, $\mathrm{H}$., et al., Black silicon solar cells with interdigitated back-contacts achieve $22.1 \%$ efficiency. Nat Nano, 2015. 10(7): p. 624-628. 


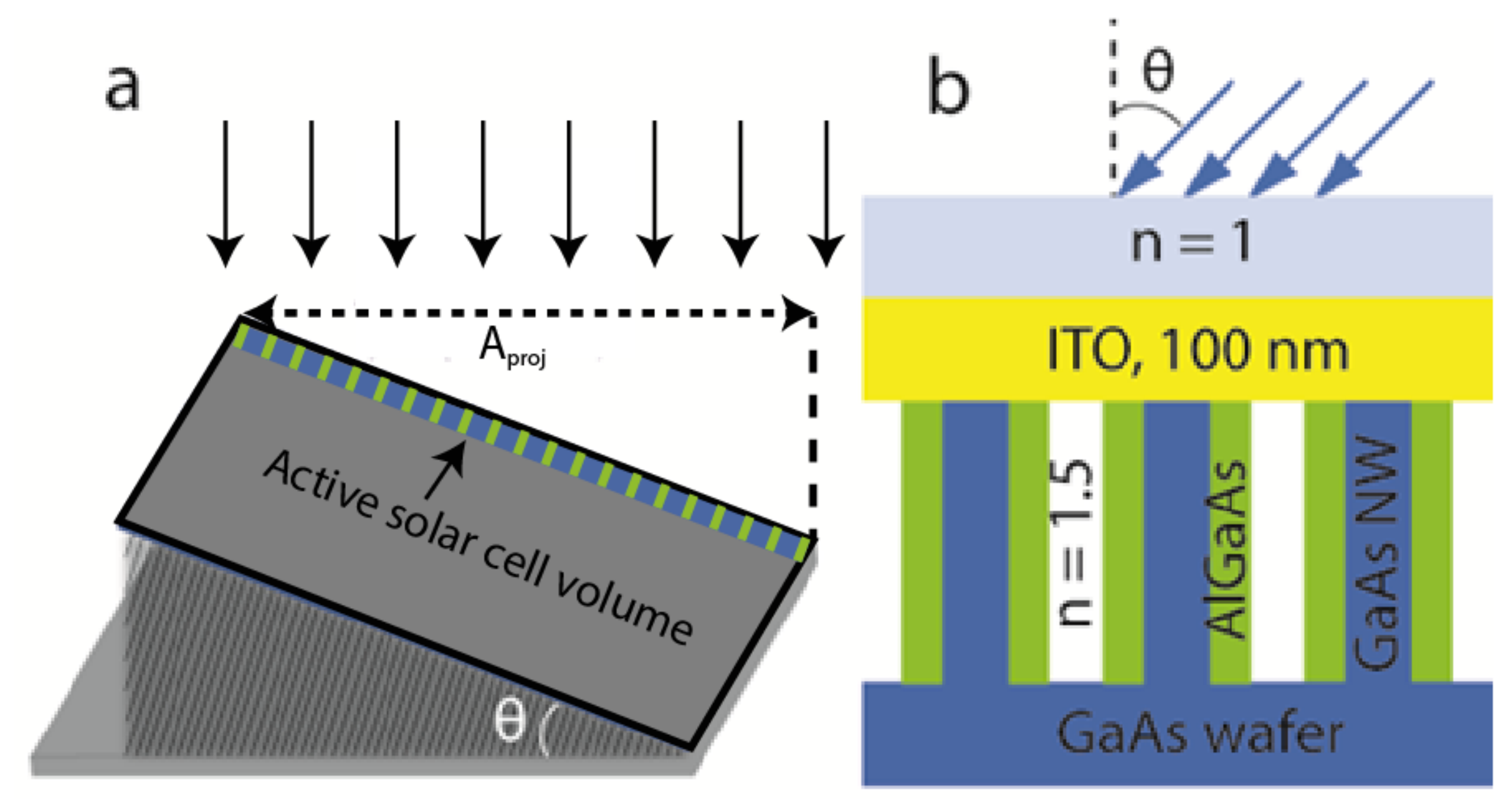




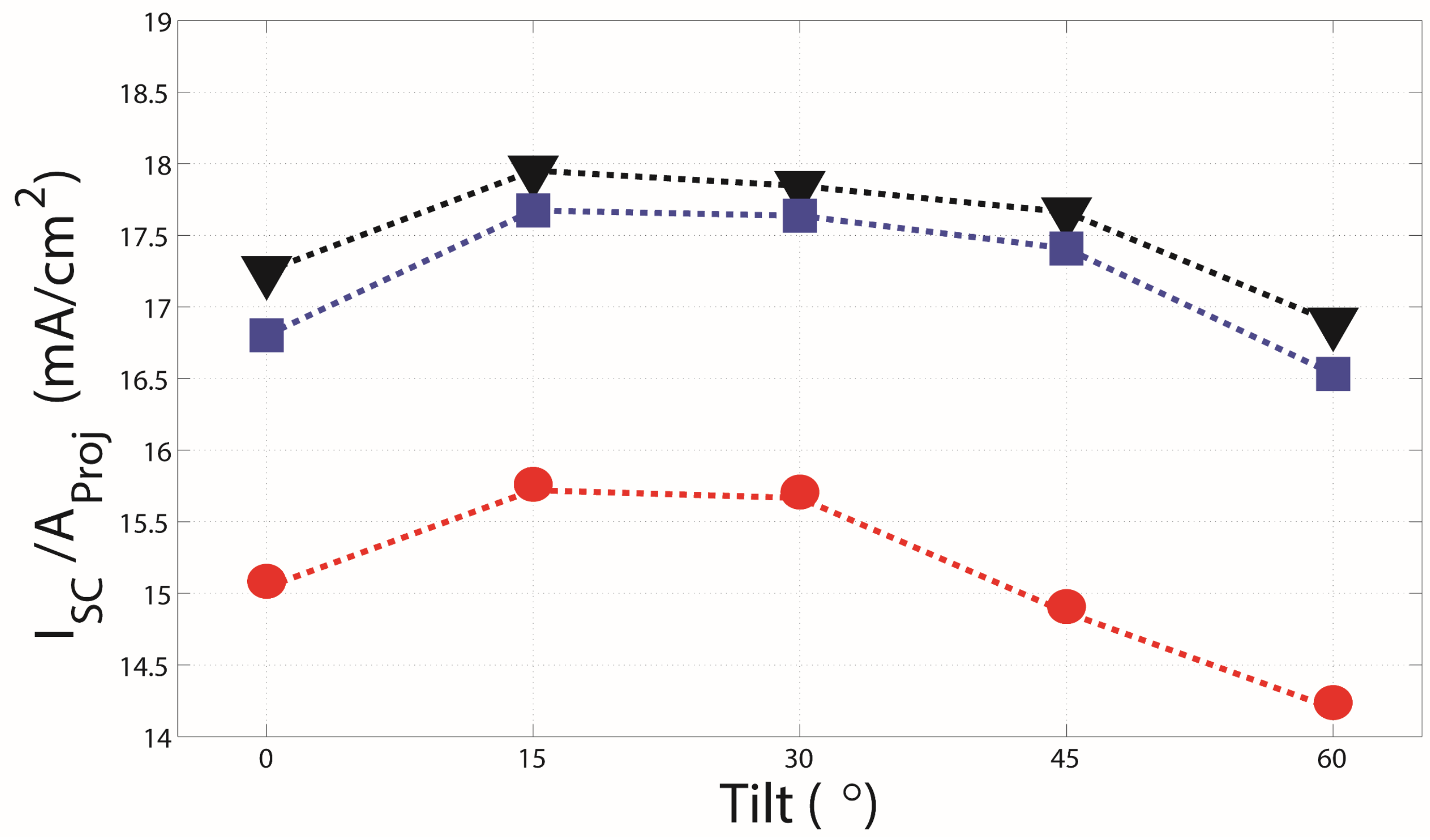

Fig2_V3 


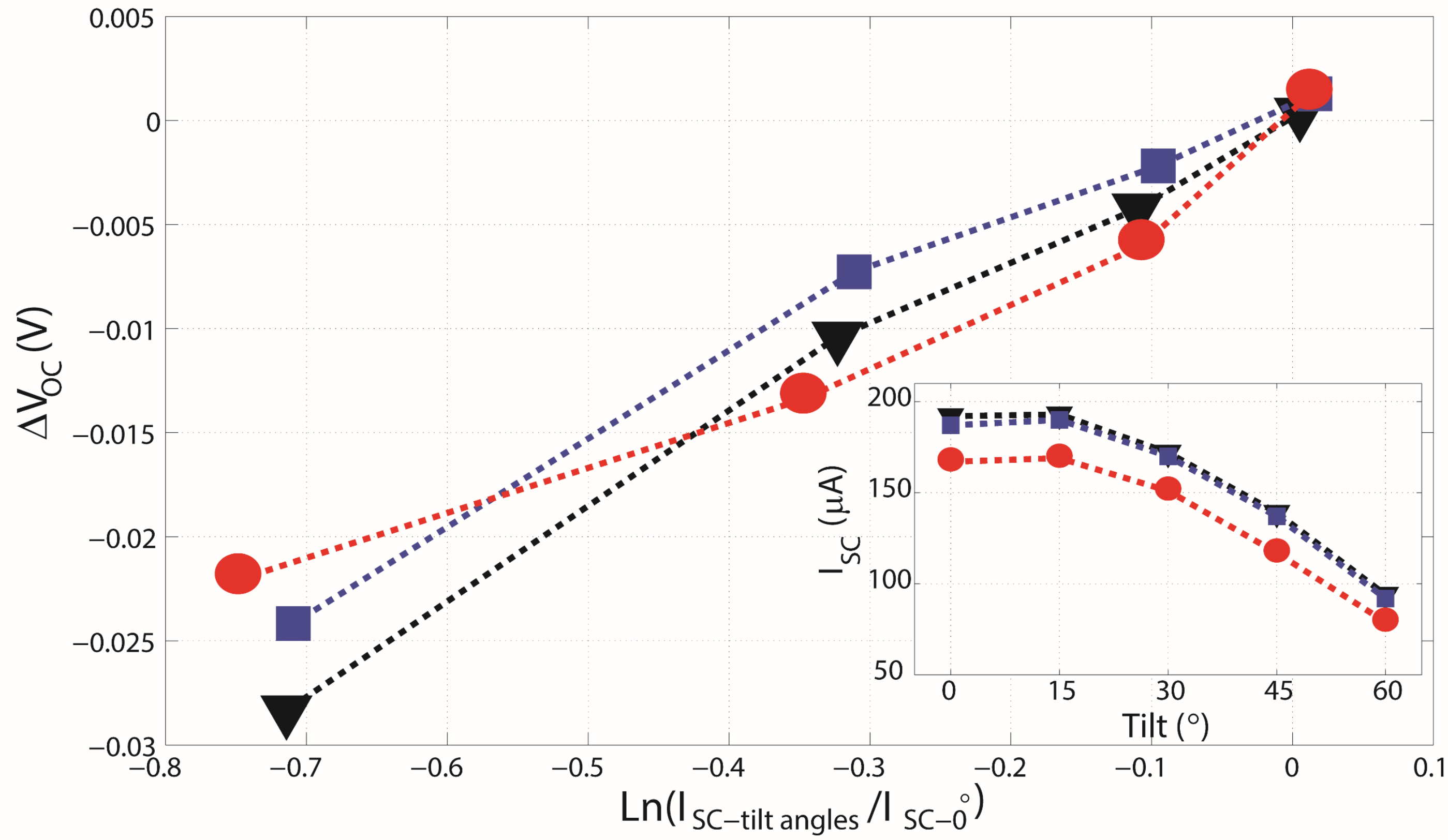

Fig3_V2 

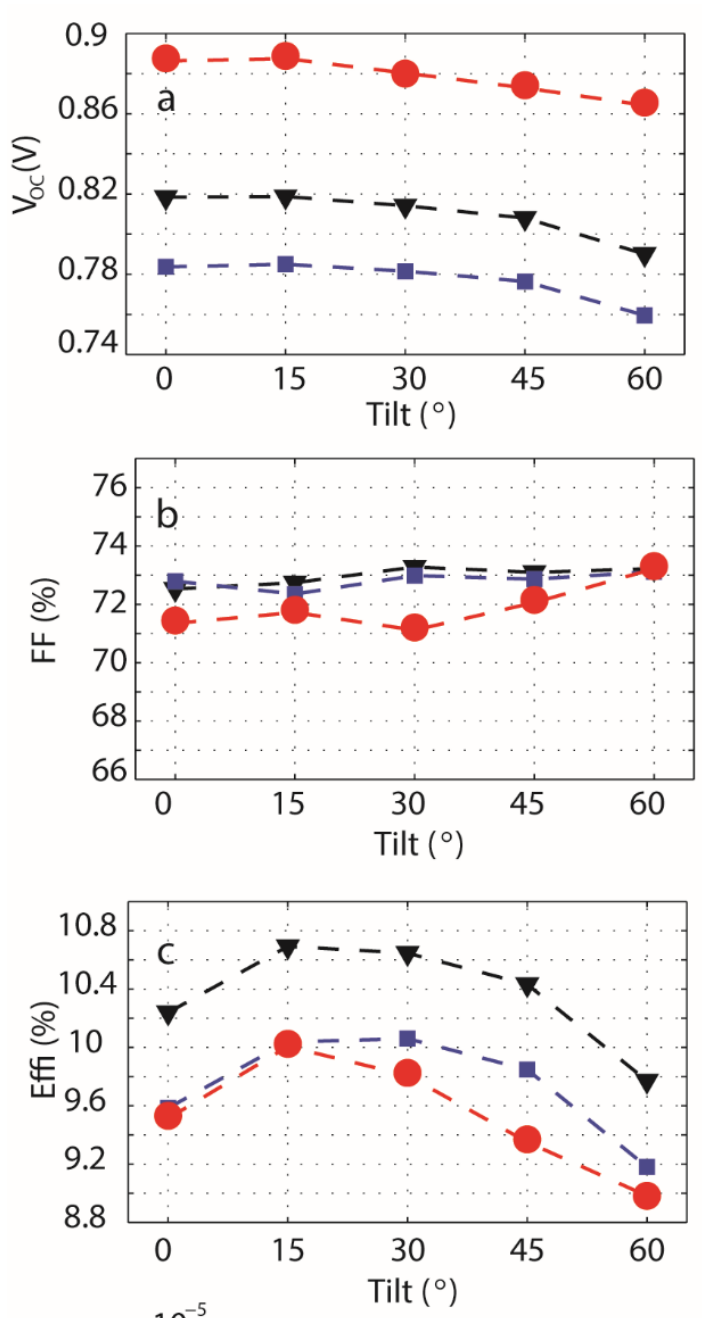

Fig4_V3

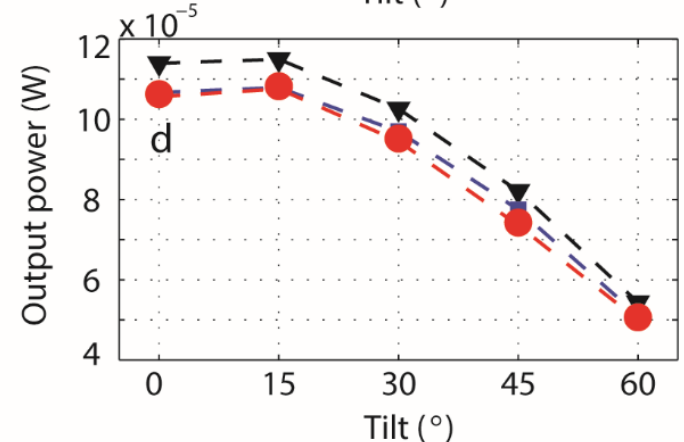




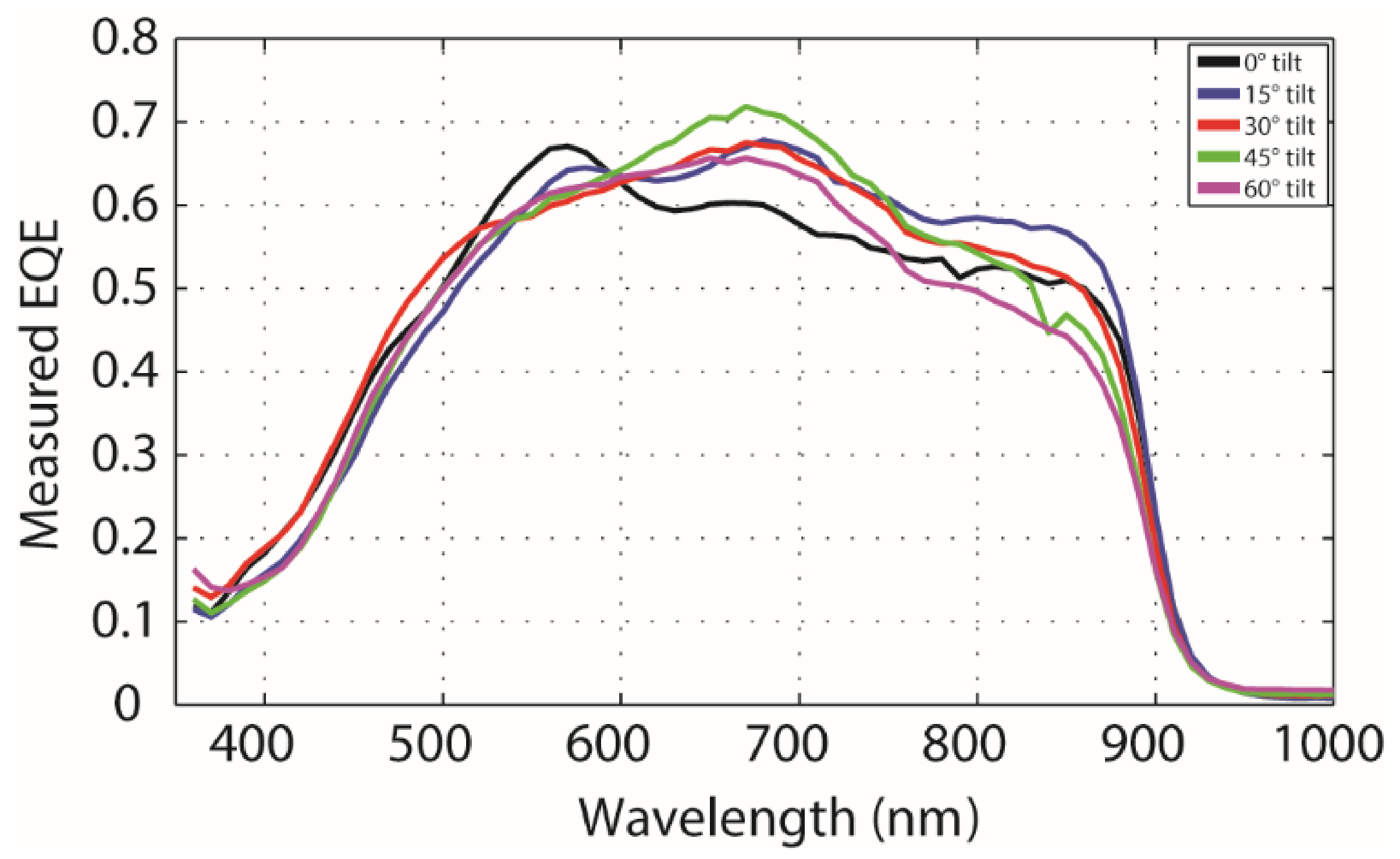

Fig5_V2 

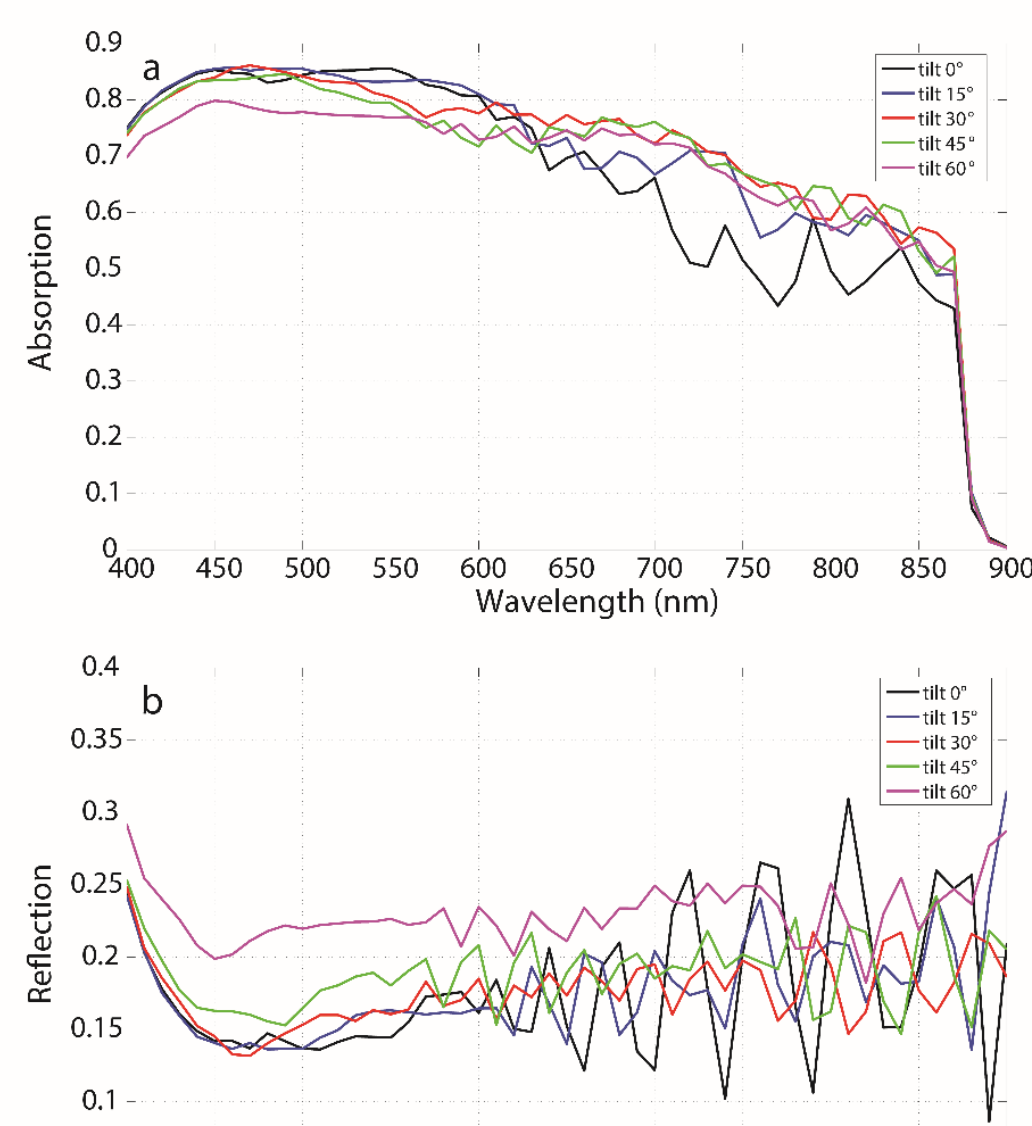

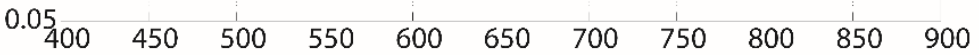

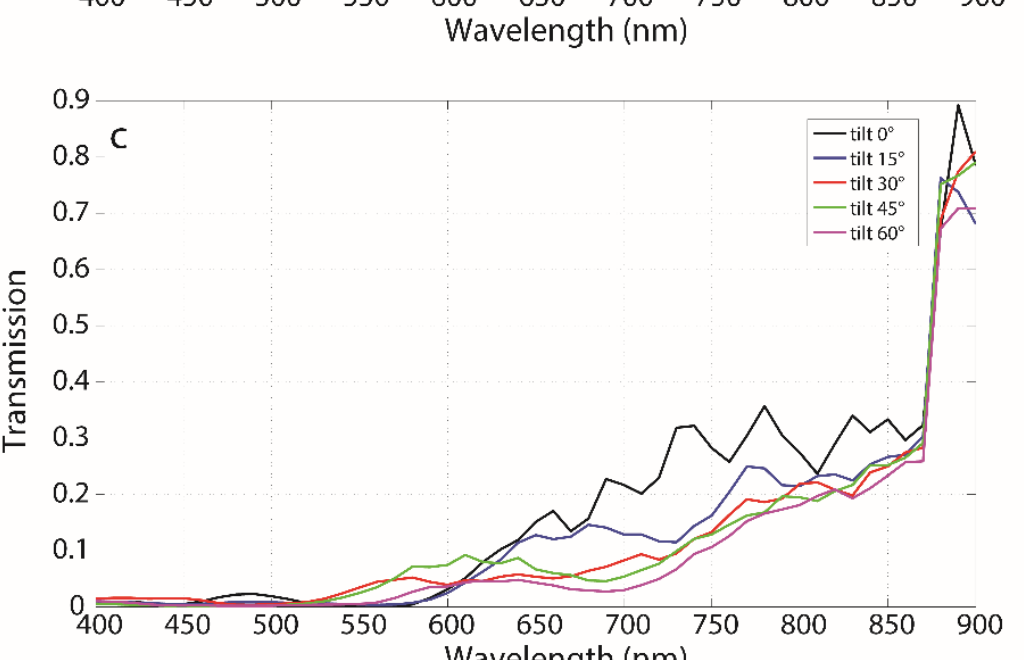

Fig6_V2 


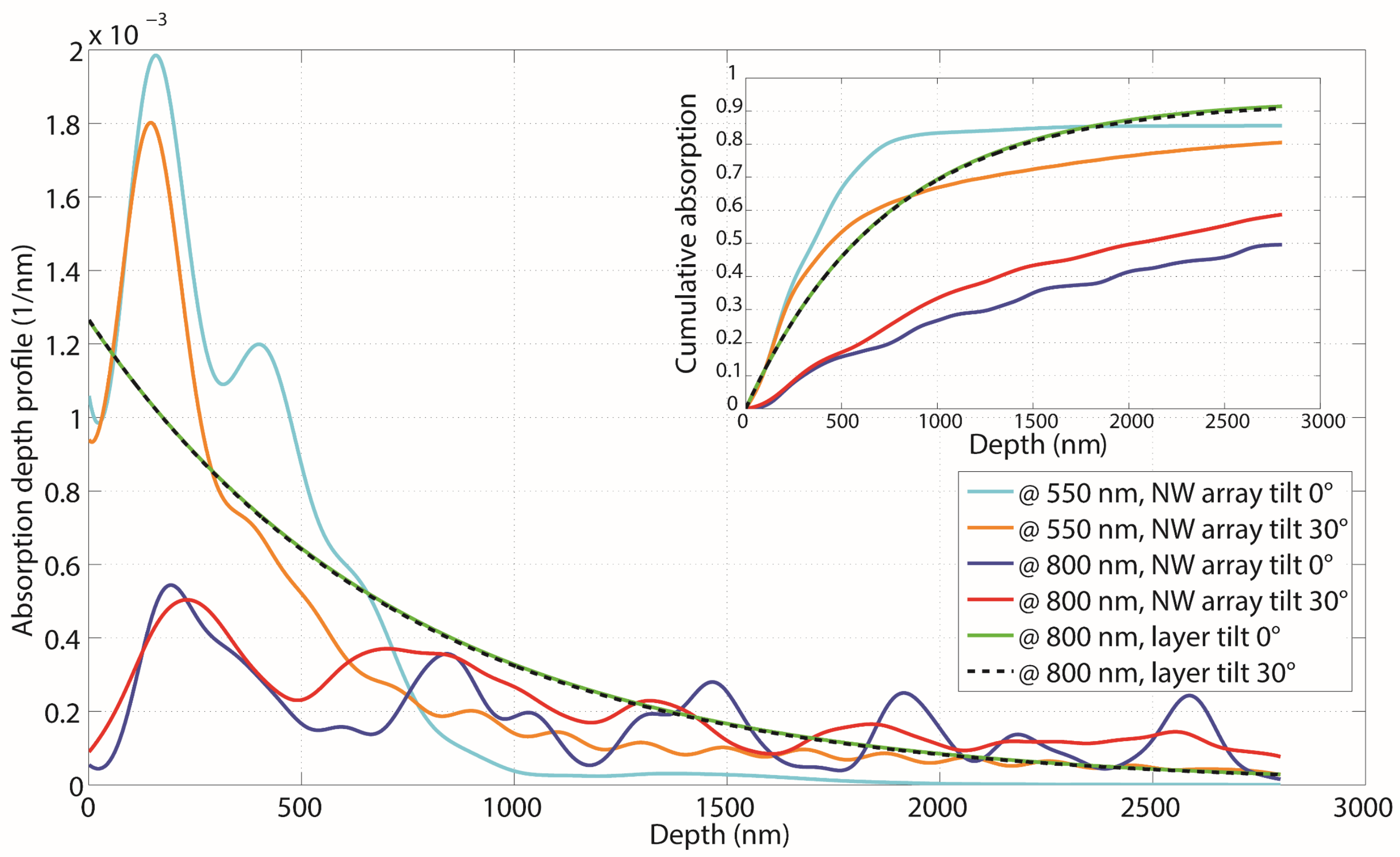

Fig7_V3 


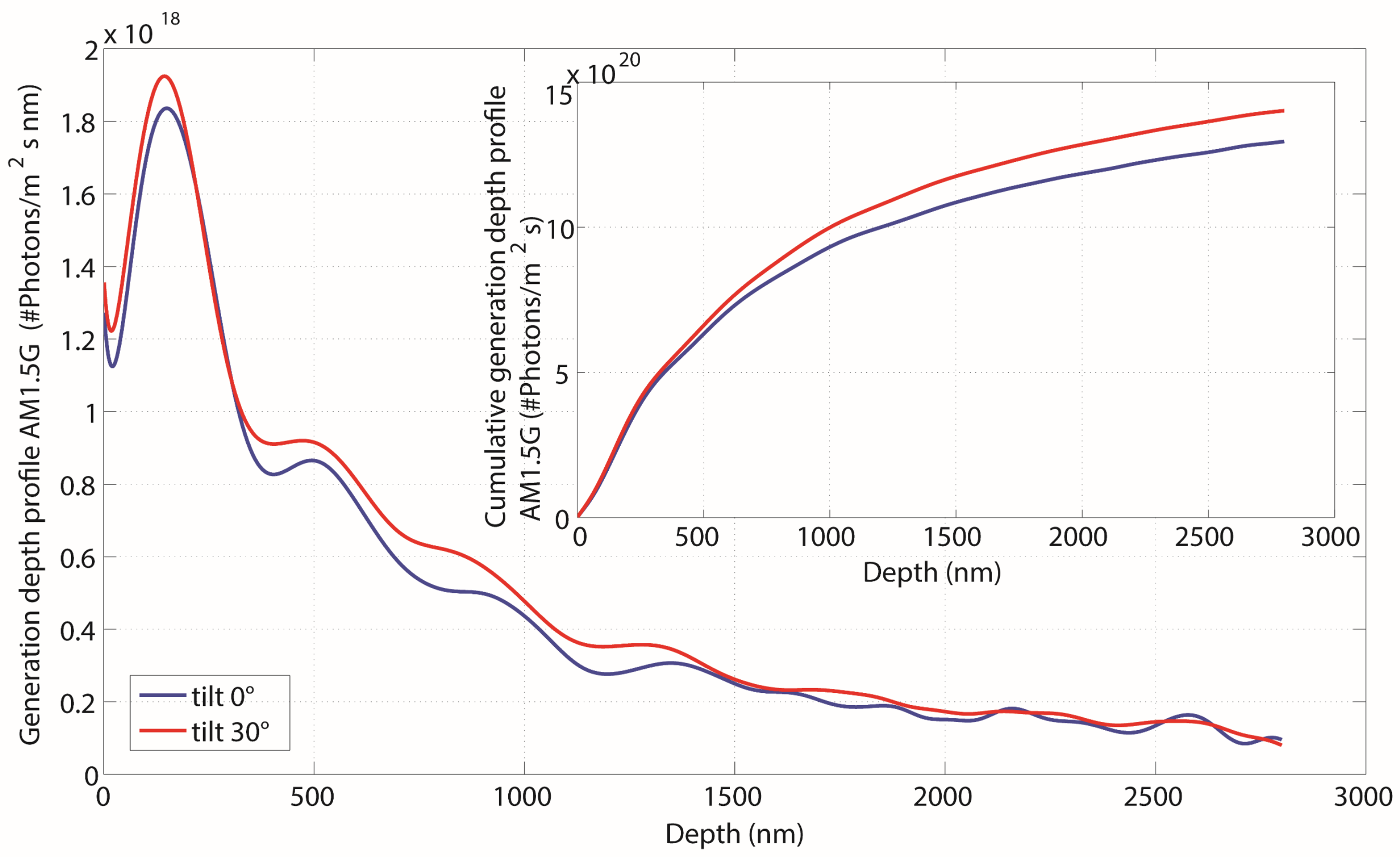

Fig8_V3 

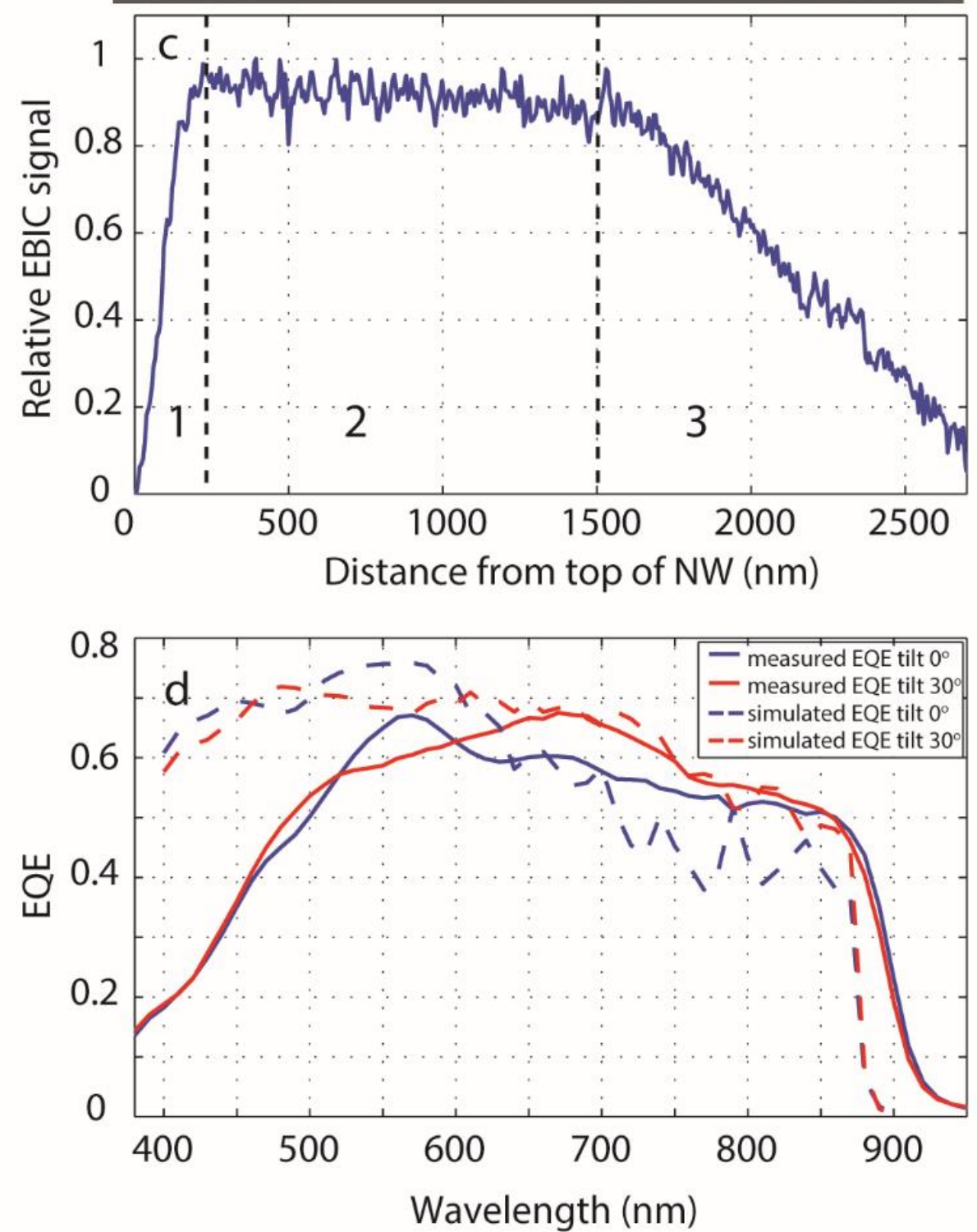